\title{
Distortionless Lossy Transmission Lines Terminated by in Series Connected RCL-Loads
}

\author{
Vasil G. Angelov ${ }^{1}$, Marin Hristov ${ }^{2}$ \\ ${ }^{1}$ Department of Mathematics, University of Mining and Geology, Sofia, Bulgaria \\ ${ }^{2}$ Department of Microelectronics, Technical University of Sofia, Sofia, Bulgaria \\ E-mail: angelov@mgu.bg,mhristov@ecad.tu-sofia.bg \\ Received July 29, 2011; revised August 26, 2011; accepted September 4, 2011
}

\begin{abstract}
The paper deals with a lossy transmission line terminated at both ends by non-linear RCL elements. The mixed problem for the hyperbolic system, describing the transmission line, to an initial value problem for a neutral equation is reduced. Sufficient conditions for the existence and uniqueness of periodic regimes are formulated. The proof is based on the finding out of suitable operator whose fixed point is a periodic solution of the neutral equation. The method has a good rate of convergence of the successive approximations even for high frequencies.
\end{abstract}

Keywords: Lossy Transmission Line, $R C L$-Nonlinear Loads, Fixed Point, Periodic Solution

\section{Introduction}

The principal importance of transmission lines investigations has been discussed in many papers (cf. for instance [1-8]). In a previous paper [9] we have investigated lossless transmission lines terminated by in series connected RCL-loads. In [10] we have considered a lossy transmission line terminated by a resistive load with exponential $V-I$ characteristic. In [11] we have considered periodic regimes for lossy transmission lines terminated by parallel connected $R C L$-loads. Here we investigate lossy transmission lines terminated at both ends by in series connected $R C L$-loads but in contrast of [11] the capacitive element has a nonlinear $V$ - $C$ characteristic. Unlike of the usually accepted approach (cf. for instance $[12,13])$ we consider first order hyperbolic system instead of the Telegrapher's equation derived from it. First we reduce the mixed problem for the hyperbolic system to an initial value problem for neutral system of equations on the boundary [14]. Extending ideas from [1517] we introduce operators whose fixed points are periodic solutions of the neutral system. Our treatment is based on the fixed point method (cf. [18]). All derivation are performed under assumption

$$
R / L=G / C \quad(R \neq 0, G \neq 0) .
$$

The last condition is known as Heaviside one and it implies that the waves propagate without distortion.

We would like to mention the advantages of our method in comparison of the other used ones: lumped element method, finite element method and finitedifference time-domain method (cf. for instance [19-21]). If we use numerical methods we have to keep one and same accuracy. But here we consider nonlinearities of polynomial and transcendental type (for exponential ones cf. [10]). For such "bad" nonlinearities (cf. [2]) there are examples showing that if we want to keep the same accuracy it should be reduced step thousands of times. Here we obtain (even though approximate) an analytical solution for voltage and current beginning with simple initial approximations.

We proceed from the system:

$$
\begin{gathered}
C \frac{\partial u(x, t)}{\partial t}+\frac{\partial i(x, t)}{\partial x}+G u(x, t)=0, \\
L \frac{\partial i(x, t)}{\partial t}+\frac{\partial u(x, t)}{\partial x}+R i(x, t)=0, \\
(x, t) \in \Pi=\left\{(x, t) \in R^{2}:(x, t) \in[0, \Lambda] \times[0, \infty)\right\}, \\
u(x, 0)=u_{0}(x), i(x, 0)=i_{0}(x), x \in[0, \Lambda]
\end{gathered}
$$

where $L, C, R$ and $G$ are prescribed specific parameters of the line and $\Lambda>0$ is its length. Here the current $i(x, t)$ and voltage $u(x, t)$ are unknown functions. The initial conditions for the foregoing system (1) are prescribed functions $u_{0}(x), i_{0}(x)$. The boundary conditions can be derived from the loads and sources at the ends of the line (cf. Figure 1). 

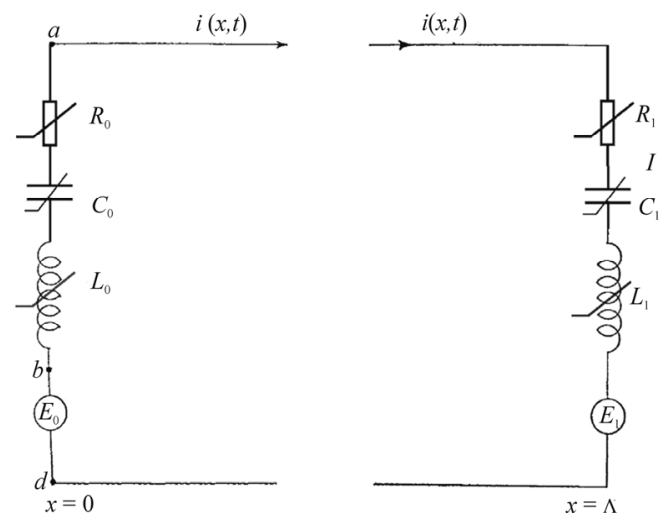

Figure 1. Lossy transmission line terminated by nonlinear RCL-loads.

In view of the Kirchoff's voltage law for the voltages between the nodes $a, b$ and $d$ for $x=0$ we obtain:

$$
-u(0, t)=-u_{a d}=u_{b a}-u_{b d}=u_{b a}-E_{0}(t),
$$

where $E_{0}(t)$ is the source voltage. The voltage $u_{b a}$ is:

$$
u_{b a}=u_{R_{0}}+u_{C_{0}}+u_{\Psi_{0}}=R_{0}(i(0, t))+u_{C_{0}}+\frac{\mathrm{d} \Psi_{0}}{\mathrm{~d} t} .
$$

To calculate the voltage of the condenser $C_{0}$ we proceed from the relation (assuming

$$
\begin{gathered}
\left.u_{C_{0}}(T) \equiv u(T)=0\right), \\
i=\frac{\mathrm{d} q}{\mathrm{~d} t}=\frac{\mathrm{d}\left(C_{0}(u) \cdot u\right)}{\mathrm{d} t} \Rightarrow \int_{T}^{t} i(x, \tau) \mathrm{d} \tau=C_{0}(u) \cdot u \equiv \bar{C}_{0}(u)
\end{gathered}
$$

or

$$
u_{C_{0}}=u(0, t)=\bar{C}_{0}^{-1}\left(\int_{T}^{t} i(0, \tau) \mathrm{d} \tau\right) .
$$

To calculate the voltage of the inductor $L_{0}$ we proceed from

$$
u_{\Psi_{0}}=\frac{\mathrm{d} \Psi_{0}}{\mathrm{~d} t}=\left(L_{0}(i) \cdot i\right)=\left(i \frac{\mathrm{d} L_{0}(i)}{\mathrm{d} i}+L_{0}(i)\right) \frac{\mathrm{d} i}{\mathrm{~d} t} .
$$

Therefore the first boundary condition is:

$$
\begin{aligned}
u_{b a}= & R_{0}(i(0, t))+\bar{C}_{0}^{-1}\left(\int_{T}^{t} i(0, \tau) \mathrm{d} \tau\right) \\
& +\left[i(0, t) \frac{\mathrm{d} L_{0}(i(0, t))}{\mathrm{d} i}+L_{0}(i(0, t))\right] \frac{\mathrm{d} i(0, t)}{\mathrm{d} t}
\end{aligned}
$$

or

$$
\begin{aligned}
u(0, t)= & E_{0}(t)-R_{0}(i(0, t))-\bar{C}_{0}^{-1}\left(\int_{T}^{t} i(0, \tau) \mathrm{d} \tau\right) \\
& -\left[i(0, t) \frac{\mathrm{d} L_{0}(i(0, t))}{\mathrm{d} i}+L_{0}(i(0, t))\right] \frac{\mathrm{d} i(0, t)}{\mathrm{d} t} .
\end{aligned}
$$

Analogously for $x=\Lambda \quad$ (cf. Figure 1) we obtain

$$
\begin{aligned}
u(\Lambda, t)= & E_{1}(t)+R_{1}(i(\Lambda, t))+\bar{C}_{1}^{-1}\left(\int_{T}^{t} i(\Lambda, t) \mathrm{d} \tau\right) \\
& +\left[i(\Lambda, t) \frac{\mathrm{d} L_{1}(i(\Lambda, t))}{\mathrm{d} i}+L_{1}(i(\Lambda, t))\right] \frac{\mathrm{d} i(\Lambda, t)}{\mathrm{d} t} .
\end{aligned}
$$

Here $R_{1}(),. C_{1}($.$) and L_{1}($.$) are characteristics (in$ general, nonlinear functions) of $R C L$-loads at right end.

Now we are able to formulate the initial-boundary (mixed) value problem for the hyperbolic transmission line system of equations: to find a solution $(u(x, t), i(x, t))$ of the hyperbolic system (1) for $(x, t) \in \Pi=\left\{(x, t) \in R^{2}: 0 \leq x \leq \Lambda, t \geq 0\right\}$, satisfying the initial conditions

$$
u(x, 0)=u_{0}(x), i(x, 0)=i_{0}(x) \text { for } x \in[0, \Lambda]
$$

and the boundary conditions

$$
\begin{aligned}
u(0, t)= & E_{0}(t)-R_{0}(i(0, t))-\bar{C}_{0}^{-1}\left(\int_{T}^{t} i(0, \tau) \mathrm{d} \tau\right) \\
& -\left[i(0, t) \frac{\mathrm{d} L_{0}(i(0, t))}{\mathrm{d} i}+L_{0}(i(0, t))\right] \frac{\mathrm{d} i(0, t)}{\mathrm{d} t}, \\
u(\Lambda, t)= & E_{1}(t)+R_{1}(i(\Lambda, t))+\bar{C}_{1}^{-1}\left(\int_{T}^{t} i(\Lambda, t) \mathrm{d} \tau\right) \\
& +\left[i(\Lambda, t) \frac{\mathrm{d} L_{1}(i(\Lambda, t))}{\mathrm{d} i}+L_{1}(i(\Lambda, t))\right] \frac{\mathrm{d} i(\Lambda, t)}{\mathrm{d} t}
\end{aligned}
$$

where $u_{0}(x), i_{0}(x), E_{k}(t), R_{k}(),. C_{k}($.$) and L_{k}($. $(k=0,1)$ are prescribed functions.

So the system (1) and conditions (6) - (8) form a mixed problem for a lossy transmission line equations.

\section{Reducing the Mixed Problem for the Transmission Line System to an Initial Value Problem for a Nonlinear Neutral System}

First we present system (1) in matrix form:

$$
A_{1} U_{t}+A_{2} U_{x}+A_{3} U=0\left(U_{t} \equiv \frac{\partial U}{\partial t}, U_{x} \equiv \frac{\partial U}{\partial x}\right)
$$

where $A_{1}=\left[\begin{array}{cc}C & 0 \\ 0 & L\end{array}\right], A_{2}=\left[\begin{array}{ll}0 & 1 \\ 1 & 0\end{array}\right], A_{3}=\left[\begin{array}{cc}G & 0 \\ 0 & R\end{array}\right]$,

$$
U=\left[\begin{array}{l}
u \\
i
\end{array}\right], U_{t}=\left[\begin{array}{l}
u_{t} \\
i_{t}
\end{array}\right], U_{x}=\left[\begin{array}{l}
u_{x} \\
i_{x}
\end{array}\right] .
$$

Since $\left|A_{1}\right|=L C \neq 0$, then multiplying Equation (9) by $A_{1}^{-1}$ we obtain 


$$
U_{t}+A U_{x}+B U=0
$$

where $A=\left[\begin{array}{lr}0 & 1 / C \\ 1 / L & 0\end{array}\right], \quad B=\left[\begin{array}{ll}G / C & 0 \\ 0 & R / L\end{array}\right]$. In order to transform the matrix $A=A_{1}^{-1} A_{2}=\left[\begin{array}{lr}0 & 1 / C \\ 1 / L & 0\end{array}\right]$ in a diagonal form we solve the characteristic equation:

$$
\left|\begin{array}{cc}
-\lambda & 1 / C \\
1 / L & -\lambda
\end{array}\right|=0
$$

whose roots are $\lambda_{1}=1 / \sqrt{L C}$ and $\lambda_{2}=-1 / \sqrt{L C}$. Denote the matrix formed by eigenvectors by

$$
H=\left[\begin{array}{rr}
\sqrt{C} & \sqrt{L} \\
-\sqrt{C} & \sqrt{L}
\end{array}\right]
$$

and its inverse one- -by

If we denote by

$$
H^{-1}=\frac{1}{2}\left[\begin{array}{cc}
1 / \sqrt{C} & -1 / \sqrt{C} \\
1 / \sqrt{L} & 1 / \sqrt{L}
\end{array}\right] .
$$

$$
A^{c a n}=\left[\begin{array}{cc}
1 / \sqrt{L C} & 0 \\
0 & -1 / \sqrt{L C}
\end{array}\right],
$$

then $A^{c a n}=H A H^{-1}$.

Introduce new variables $Z=H U\left(\right.$ or $\left.U=H^{-1} Z\right)$ :

$$
Z=\left[\begin{array}{l}
V(x, t) \\
I(x, t)
\end{array}\right], U=\left[\begin{array}{l}
u(x, t) \\
i(x, t)
\end{array}\right] .
$$

Then

$$
\begin{aligned}
& \mid \begin{array}{l}
V(x, t)=\sqrt{C} u(x, t)+\sqrt{L} i(x, t) \\
I(x, t)=-\sqrt{C} u(x, t)+\sqrt{L} i(x, t),
\end{array} \\
& \mid \begin{array}{l}
u(x, t)=V(x, t) / 2 \sqrt{C}-I(x, t) / 2 \sqrt{C} \\
i(x, t)=V(x, t) / 2 \sqrt{L}+I(x, t) / 2 \sqrt{L} .
\end{array}
\end{aligned}
$$

Replacing $U=H^{-1} Z$ in Equation (10) we obtain

$$
\frac{\partial\left(H^{-1} Z\right)}{\partial t}+A \frac{\partial\left(H^{-1} Z\right)}{\partial x}+B\left(H^{-1} Z\right)=0 .
$$

Since $H^{-1}$ is a constant matrix we have:

$$
H^{-1} Z_{t}+\left(A H^{-1}\right) Z_{x}+\left(B H^{-1}\right) Z=0 .
$$

After multiplication from the left by $H$ we obtain

$$
\begin{aligned}
& Z_{t}+H\left(A H^{-1}\right) Z_{x}+H\left(B H^{-1}\right) Z=0, \text { i.e. } \\
& Z_{t}+A^{c a n} Z_{x}+\left(H B H^{-1}\right) Z=0 .
\end{aligned}
$$

But

$$
H B H^{-1}=\frac{1}{2}\left[\begin{array}{lc}
(G / C)+(R / L) & (-G / C)+(R / L) \\
(-G / C)+(R / L) & (G / C)+(R / L)
\end{array}\right]
$$

and then

$$
\begin{aligned}
& {\left[\begin{array}{c}
V_{t} \\
I_{t}
\end{array}\right]+\left[\begin{array}{cc}
1 / \sqrt{L C} & 0 \\
0 & -1 / \sqrt{L C}
\end{array}\right]\left[\begin{array}{l}
V_{x} \\
I_{x}
\end{array}\right]} \\
& +\frac{1}{2}\left[\begin{array}{ll}
(G / C)+(R / L) & (-G / C)+(R / L) \\
(-G / C)+(R / L) & (G / C)+(R / L)
\end{array}\right]\left[\begin{array}{l}
V \\
I
\end{array}\right]=\left[\begin{array}{l}
0 \\
0
\end{array}\right] .
\end{aligned}
$$

We consider distortionless lossy transmission lines that means the following Heaviside condition is fulfilled:

$$
R / L=G / C .
$$

Then $H_{B H}{ }^{-1}$ can be simplified and the last system becomes:

$$
\left[\begin{array}{c}
V_{t} \\
I_{t}
\end{array}\right]+\left[\begin{array}{cc}
1 / \sqrt{L C} & 0 \\
0 & -1 / \sqrt{L C}
\end{array}\right]\left[\begin{array}{l}
V_{x} \\
I_{x}
\end{array}\right]+\left[\begin{array}{cr}
R / L & 0 \\
0 & R / L
\end{array}\right]\left[\begin{array}{l}
V \\
I
\end{array}\right]=\left[\begin{array}{l}
0 \\
0
\end{array}\right]
$$

or

$$
\begin{aligned}
& V_{t}+(1 / \sqrt{L C}) V_{x}+(R / L) V=0 \\
& I_{t}-(1 / \sqrt{L C}) I_{x}+(R / L) I=0 .
\end{aligned}
$$

The new initial conditions we obtain from conditions (6) and system (12):

$$
\begin{aligned}
V(x, 0) & =\sqrt{C} u(x, 0)+\sqrt{L} i(x, 0) \\
& =\sqrt{C} u_{0}(x)+\sqrt{L} i_{0}(x) \equiv V_{0}(x), x \in[0, \Lambda], \\
I(x, 0) & =-\sqrt{C} u(x, 0)+\sqrt{L} i(x, 0) \\
& =-\sqrt{C} u_{0}(x)+\sqrt{L} i_{0}(x) \equiv I_{0}(x), x \in[0, \Lambda] .
\end{aligned}
$$

Further on we set

$$
\begin{gathered}
W(x, t)=e^{(R / L) t} V(x, t), J(x, t)=e^{(R / L) t} I(x, t) \\
\Leftrightarrow V(x, t)=e^{-(R / L) t} W(x, t), I(x, t)=e^{-(R / L) t} J(x, t) .
\end{gathered}
$$

Then system (14) can be written in the form:

$$
W_{t}+v W_{x}=0, \quad J_{t}-v J_{x}=0 \quad(v=1 / \sqrt{L C}) .
$$

The initial conditions (15), (16) remain the same ones: $W(0, t)=V(0, t)=V_{0}(x), J(0, t)=I(0, t)=I_{0}(x)$.

From system (12) and denotation (17) we obtain

$$
\begin{aligned}
& u(x, t)=[W(x, t)-J(x, t)] e^{-(R / L) t} /(2 \sqrt{C}), \\
& i(x, t)=[W(x, t)+J(x, t)] e^{-(R / L) t} /(2 \sqrt{L})
\end{aligned}
$$

and then

$$
\begin{aligned}
& W(x, t)=e^{(R / L) t} \sqrt{C} u(x, t)+e^{(R / L) t} \sqrt{L} i(x, t), \\
& J(x, t)=-e^{(R / L) t} \sqrt{C} u(x, t)+e^{(R / L) t} \sqrt{L} i(x, t) .
\end{aligned}
$$


Replacing in system (19) $x=0$ we have

$$
\begin{aligned}
& u(0, t)=[W(0, t)-J(0, t)] e^{-(R / L) t} /(2 \sqrt{C}), \\
& i(0, t)=[W(0, t)+J(0, t)] e^{-(R / L) t} /(2 \sqrt{L})
\end{aligned}
$$

and replacing $x=\Lambda-$

$$
\begin{aligned}
& u(\Lambda, t)=[W(\Lambda, t)-J(\Lambda, t)] e^{-(R / L) t} /(2 \sqrt{C}), \\
& i(\Lambda, t)=[W(\Lambda, t)+J(\Lambda, t)] e^{-(R / L) t} /(2 \sqrt{L}) .
\end{aligned}
$$

Now replace expressions (20) into the first boundary condition and obtain:

$$
\begin{aligned}
& {[W(0, t)-J(0, t)] e^{-(R / L) t} /(2 \sqrt{C}) } \\
= & E_{0}(t)-R_{0}\left((W(0, t)+J(0, t)) e^{-(R / L) t} /(2 \sqrt{L})\right) \\
& -\bar{C}_{0}^{-1}\left(\int_{T}^{t}[W(0, \tau)+J(0, \tau)] e^{-(R / L) t} /(2 \sqrt{L}) \mathrm{d} \tau\right) \\
& -\left[(i(0, t)) \frac{\mathrm{d} L_{0}(i(0, t))}{\mathrm{d} i}+L_{0}(i(0, t))\right] \\
& \times \frac{\mathrm{d}}{\mathrm{d} t}\left((W(0, t)+J(0, t)) e^{-(R / L) t} /(2 \sqrt{L})\right) .
\end{aligned}
$$

Replacing expressions (21) into the second boundary condition we obtain the following equation

$$
\begin{aligned}
& {[W(\Lambda, t)-J(\Lambda, t)] e^{-(R / L) t} /(2 \sqrt{C}) } \\
= & E_{1}(t)+R_{1}\left((W(\Lambda, t)+J(\Lambda, t)) e^{-(R / L) t} /(2 \sqrt{L})\right) \\
& +\bar{C}_{1}^{-1}\left(\int_{T}^{t}[W(\Lambda, s)+J(\Lambda, s)] e^{-(R / L) s} /(2 \sqrt{L}) \mathrm{d} s\right) \\
& +\left[i(\Lambda, t) \frac{\mathrm{d} L_{1}(i(\Lambda, t))}{\mathrm{d} i}+L_{1}(i(\Lambda, t))\right] \\
& \times \frac{\mathrm{d}}{\mathrm{d} t}\left((W(\Lambda, t)+J(\Lambda, t)) e^{-(R / L) t} /(2 \sqrt{L})\right) .
\end{aligned}
$$

Introduce denotations

$$
\begin{aligned}
\tilde{L}_{0}(t)= & i(0, t) \frac{\mathrm{d} L_{0}(i(0, t))}{\mathrm{d} i}+L_{0}(i(0, t)) \\
= & \left((W(0, t)+J(0, t)) e^{-(R / L) t} /(2 \sqrt{L})\right) \frac{\mathrm{d} L_{0}(i(0, t))}{\mathrm{d} i} \\
& +L_{0}\left((W(0, t)+J(0, t)) e^{-(R / L) t} /(2 \sqrt{L})\right), \\
\tilde{L}_{1}(t)= & i(\Lambda, t) \frac{\mathrm{d} L_{1}(i(\Lambda, t))}{\mathrm{d} i}+L_{1}(i(\Lambda, t))
\end{aligned}
$$

$$
\begin{aligned}
= & \left((W(\Lambda, t)+J(\Lambda, t)) e^{-(R / L) t} /(2 \sqrt{L})\right) \frac{\mathrm{d} L_{1}(i(\Lambda, t))}{\mathrm{d} i} \\
& +L_{1}\left((W(\Lambda, t)+J(\Lambda, t)) e^{-(R / L) t} /(2 \sqrt{L})\right) .
\end{aligned}
$$

We assume that the unknown functions are $W(\Lambda, t)$ $=W(t), J(0, t)=J(t)$. An integration along the charateristics yields $W(\Lambda, t+T)=W(0, t), J(\Lambda, t)=J(0, t+T)$ and then Equations (22) and (23) become

$$
\begin{aligned}
& (W(0, t+T)-J(0, t)) e^{-(R / L) t} /(2 \sqrt{C}) \\
= & E_{0}(t)-R_{0}\left((W(0, t+T)-J(0, t)) e^{-(R / L) t} /(2 \sqrt{C})\right) \\
& -\bar{C}_{0}^{-1}\left(\int_{T}^{t}\left((W(0, t+T)+J(0, \tau)) e^{-(R / L) \tau} /(2 \sqrt{L})\right) \mathrm{d} \tau\right) \\
& -\tilde{L}_{0}(t) \frac{\mathrm{d}}{\mathrm{d} t}\left((W(0, t+T)+J(0, \tau)) e^{-(R / L) \tau} /(2 \sqrt{L})\right)
\end{aligned}
$$

and

$$
\begin{aligned}
& (W(\Lambda, t)-J(0, t+T)) e^{-(R / L) t} /(2 \sqrt{C}) \\
= & E_{1}(t)+R_{1}\left((W(\Lambda, t)+J(0, t+T)) e^{-(R / L) t} /(2 \sqrt{L})\right) \\
+ & \bar{C}_{1}^{-1}\left(\int_{T}^{t}\left([W(\Lambda, \tau)+J(0, \tau+T)] e^{-(R / L) \tau} /(2 \sqrt{L})\right) \mathrm{d} \tau\right. \\
& \left.+\tilde{L}_{1}(t) \frac{\mathrm{d}}{\mathrm{d} t}\left((W(\Lambda, t)+J(0, t+T)) e^{-(R / L) t} /(2 \sqrt{L})\right)\right) .
\end{aligned}
$$

Then we put $t+T \equiv t$ and change the variables in the integrals:

$$
\begin{aligned}
& (W(t)-J(t-T)) e^{-(R / L)(t-T)} /(2 \sqrt{C}) \\
= & E_{0}(t-T)-R_{0}\left((W(t)+J(t-T)) e^{-(R / L)(t-T)} /(2 \sqrt{L})\right) \\
& -\bar{C}_{0}^{-1} \int_{T}^{t}\left((W(\theta)+J(\theta-T)) e^{-(R / L)(\theta-T)} /(2 \sqrt{L})\right) \mathrm{d} \theta \\
& -\tilde{L}_{0}(t-T) \frac{\mathrm{d}}{\mathrm{d} t}\left((W(t)+J(t-T)) e^{-(R / L)(t-T)} /(2 \sqrt{L})\right), \\
& (W(t-T)-J(t)) e^{-(R / L)(t-T)} /(2 \sqrt{C}) \\
= & E_{1}(t-T)+R_{1}\left((W(t-T)+J(t)) e^{-(R / L)(t-T)} /(2 \sqrt{C})\right) \\
& +\bar{C}_{1}^{-1} \int_{T}^{t}\left((W(\theta-T)+J(\theta)) e^{-(R / L)(\theta-T)} /(2 \sqrt{L})\right) \mathrm{d} \theta \\
& +\tilde{L}_{1}(t-T) \frac{\mathrm{d}}{\mathrm{d} t}\left((W(t-T)+J(t)) e^{-(R / L)(t-T)} /(2 \sqrt{L})\right) .
\end{aligned}
$$

To solve the above equations with respect to the derivatives $\mathrm{d} W(t) / \mathrm{d} t$ and $\mathrm{d} J(t) / \mathrm{d} t$ we have to divide the above equations into $\tilde{L}_{0}(t-T)$ and $\tilde{L}_{1}(t-T)$, respectively.

But 
$u_{\Psi_{k}}=\frac{\mathrm{d} \Psi_{k}}{\mathrm{~d} t}=\frac{\mathrm{d} \tilde{L}_{k}(i)}{\mathrm{d} t} \equiv \frac{\mathrm{d} L_{k}(i) \cdot i}{\mathrm{~d} t}=\left(i \frac{\mathrm{d} L_{k}(i)}{\mathrm{d} i}+L_{k}(i)\right) \frac{\mathrm{d} i}{\mathrm{~d} t}$

$(k=0,1)$ where $L_{k}(i)=\sum_{n=0}^{m} a_{n}^{(k)} i^{n}$. Then since

$$
\Psi_{k}(i)=i \cdot L_{k}(i)=i \cdot \sum_{n=0}^{m} a_{n}^{(k)} i^{n}=\sum_{n=0}^{m} a_{n}^{(k)} i^{n+1} .
$$

we get

$$
\begin{aligned}
\frac{\mathrm{d} \tilde{L}_{k}(i)}{\mathrm{d} i} & =i \frac{\mathrm{d} L_{k}(i)}{\mathrm{d} i}+L_{k}(i) \\
& =i \sum_{n=0}^{m} n a_{n}^{(k)} i^{n-1}+\sum_{n=0}^{m} a_{n}^{(k)} i^{n} \\
& =\sum_{n=0}^{m}(n+1) a_{n}^{(k)} i^{n},(k=0,1) .
\end{aligned}
$$

Introduce denotations

$$
\begin{aligned}
& \Pi_{0}(W, J)(t)=e^{-(R / L)(t-T)}(W(t)+J(t-T)) /(2 \sqrt{L}), \\
& \Pi_{1}(W, J)(t)=e^{-(R / L)(t-T)}(W(t-T)+J(t)) /(2 \sqrt{L}) .
\end{aligned}
$$

Therefore

$$
\begin{aligned}
\tilde{L}_{0}(t-T)= & \Pi_{0}(W, J)(t) \frac{\mathrm{d} L_{0}\left(\Pi_{0}(W, J)(t)\right)}{\mathrm{d} i} \\
& +L_{0}\left(\Pi_{0}(W, J)(t)\right) \\
= & \sum_{n=0}^{m}(n+1) a_{n}^{(0)}\left(\Pi_{0}(W, J)(t)\right)^{n}, \\
\tilde{L}_{1}(t-T)= & \Pi_{1}(W, J)(t) \frac{\mathrm{d} L_{1}\left(\Pi_{1}(W, J)(t)\right)}{\mathrm{d} i} \\
& +L_{1}\left(\Pi_{1}(W, J)(t)\right) \\
= & \sum_{n=0}^{m}(n+1) a_{n}^{(1)}\left(\Pi_{1}(W, J)(t)\right)^{n} .
\end{aligned}
$$

We have to ensure a strict positive lower bound for $\tilde{L}_{k}(t-T),(k=0,1)$. We can find an interval $|i| \leq i_{0}$ such that the inequalities

$$
\begin{aligned}
& \left|\Pi_{0}(W, J)(t)\right| \leq e^{(\mu-(R / L)) T_{0}}\left(W_{0}+J_{0} e^{-\mu T}\right) /(2 \sqrt{L}) \leq i_{0}, \\
& \left|\Pi_{1}(W, J)(t)\right| \leq e^{(\mu-(R / L)) T_{0}}\left(W_{0} e^{-\mu T}+J_{0}\right) /(2 \sqrt{L}) \leq i_{0}
\end{aligned}
$$

imply

$$
\begin{aligned}
& \tilde{L}_{k}(t-T)=\sum_{n=0}^{m}(n+1) a_{n}^{(k)}\left(\Pi_{k}(W, J)(t)\right)^{n} \geq \bar{L}_{k}>0 \\
\Rightarrow & 1 / \tilde{L}_{k}(t-T) \leq 1 / \bar{L}_{k} \leq 1 / \bar{L} \quad(k=0,1)
\end{aligned}
$$

where $1 / \bar{L}=\max \left\{1 / \bar{L}_{k}: k=0,1\right\}$.

This can be done if the polynomials have suitable properties (cf. 4. Numerical example).
We also briefly recall:

$$
i=\frac{\mathrm{d} q}{\mathrm{~d} t}=\frac{\mathrm{d}\left(C_{0}(u) \cdot u\right)}{\mathrm{d} t} \Rightarrow \int_{T}^{t} i(x, \tau) \mathrm{d} \tau=C_{0}(u) \cdot u \equiv \bar{C}_{0}(u)
$$

and

$$
u_{C_{0}}=u(0, t)=\bar{C}_{0}^{-1}\left(\int_{T}^{t} i(0, \tau) \mathrm{d} \tau\right)
$$

where $c_{k}, \Phi_{k}, h \in[2,3]$,

$$
C_{k}(u)=\frac{c_{k}}{\sqrt[h]{1-\left(u / \Phi_{k}\right)}}=\frac{c_{k} \sqrt[h]{\Phi_{k}}}{\sqrt[h]{\Phi_{k}-u}}(k=0,1)
$$

are constants and

$$
|u| \leq W_{0}<\Phi=\min \left\{\Phi_{0}, \Phi_{1}\right\} \leq(h /(h-1)) \Phi .
$$

The derivatives of the functions $\bar{C}_{k}(u)=u C_{k}(u)$ are

$$
\begin{aligned}
\frac{\mathrm{d} \bar{C}_{k}(u)}{\mathrm{d} u} & =C_{k}(u)+u \frac{\mathrm{d} C_{k}(u)}{\mathrm{d} u} \\
& =\frac{c_{k} \sqrt[h]{\Phi_{k}}\left(h \Phi_{k}-h u+u\right)}{h\left(\Phi_{k}-u\right)^{\frac{1}{h}+1}}, u \in\left[-W_{0}, W_{0}\right] .
\end{aligned}
$$

Since $\frac{\mathrm{d} \bar{C}_{k}(u)}{\mathrm{d} u}>0$ the inverse function exists for $|u|<\frac{h \Phi_{k}}{h-1}$.

Since

$$
\begin{aligned}
& \bar{C}_{k}(u)=u C_{k}(u)=\frac{c_{k} u \sqrt[h]{\Phi_{k}}}{\sqrt[h]{\Phi_{k}-u}}:\left[-W_{0}, W_{0}\right] \\
\rightarrow & {\left[\frac{-c_{k} \sqrt[h]{\Phi_{k}} W_{0}}{\sqrt[h]{\Phi_{k}+W_{0}}}, \frac{c_{k} \sqrt[h]{\Phi_{k}} W_{0}}{\sqrt[h]{\Phi_{k}-W_{0}}}\right] }
\end{aligned}
$$

then

$$
\bar{C}_{k}^{-1}(I):\left[\frac{-C_{k} \sqrt[h]{\Phi_{k}} W_{0}}{\sqrt[h]{\Phi_{k}+W_{0}}}, \frac{c_{k} \sqrt[h]{\Phi_{k}} W_{0}}{\sqrt[h]{\Phi_{k}-W_{0}}}\right] \rightarrow\left[-W_{0}, W_{0}\right],
$$

or $\left|\bar{C}_{k}^{-1}(I)\right| \leq W_{0}$.

The explicit form of the inverse function for $h=2$ is:

$$
\begin{aligned}
& c_{k} \sqrt{\Phi_{k}} u / \sqrt{\Phi_{k}-u}=I \\
\Rightarrow & c_{k}^{2} \Phi_{k} u^{2}=\Phi_{k} I^{2}-I^{2} u \\
\Rightarrow & c_{k}^{2} \Phi_{k} u^{2}+I^{2} u-\Phi_{k} I^{2}=0, \\
\bar{C}_{k}^{-1}(I) & \\
\quad= & \frac{-I^{2}+\sqrt{I^{4}+4 c_{k}^{2} \Phi_{k}^{2} I^{2}}}{2 c_{k}^{2} \Phi_{k}}(k=0,1) .
\end{aligned}
$$

We need the derivative given below (see Equation (26)) 
and the following estimates:

$$
|I| \leq \frac{c_{k} \sqrt{\Phi_{k}} W_{0}}{\sqrt{\Phi_{k}+W_{0}}}=\min \left\{\left|\frac{-c_{k} \sqrt[h]{\Phi_{k}} W_{0}}{\sqrt[h]{\Phi_{k}+W_{0}}}\right|,\left|\frac{c_{k} \sqrt[h]{\Phi_{k}} W_{0}}{\sqrt[h]{\Phi_{k}-W_{0}}}\right|\right\}
$$

and

$$
\begin{aligned}
\left|\frac{\mathrm{d} \bar{C}_{k}^{-1}(I)}{\mathrm{d} I}\right| & \leq \frac{1}{c_{k}^{2} \Phi_{k}}\left(|I|+\frac{I^{2}+4 c_{k}^{2} \Phi_{k}^{2}}{\sqrt{I^{2}+4 c_{k}^{2} \Phi_{k}^{2}}}\right) \\
& \leq \frac{1}{c_{k}^{2} \Phi_{k}}\left(|I|+\sqrt{I^{2}+4 c_{k}^{2} \Phi_{k}^{2}}\right) \\
& \leq \frac{1}{c_{k}^{2} \Phi_{k}}\left(\frac{c_{k} \sqrt{\Phi_{k}} W_{0}}{\sqrt{\Phi_{k}+W_{0}}}+\sqrt{\left(\frac{c_{k} \sqrt{\Phi_{k}} W_{0}}{\sqrt{\Phi_{k}+W_{0}}}\right)^{2}+4 c_{k}^{2} \Phi_{k}^{2}}\right) \\
& \leq \frac{2}{c_{k}} \sqrt{1+\frac{W_{0}}{\Phi_{k}}}(k=0,1) .
\end{aligned}
$$

Therefore the arguments

$$
\begin{aligned}
& \int_{T}^{t}\left([W(\theta)+J(\theta-T)] e^{-(R / L)(\theta-T)} /(2 \sqrt{L})\right) \mathrm{d} \theta, . \\
& \int_{T}^{t}\left([W(\theta-T)+J(\theta)] e^{-(R / L)(\theta-T)} /(2 \sqrt{L})\right) \mathrm{d} \theta
\end{aligned}
$$

of $\bar{C}_{0}^{-1}(),. \bar{C}_{1}^{-1}($.$) should satisfy the inequalities$

$$
\begin{aligned}
& \left|\int_{T}^{t}\left([W(\theta)+J(\theta-T)] e^{-(R / L)(\theta-T)} /(2 \sqrt{L})\right) \mathrm{d} \theta\right| \leq \frac{c_{k} \sqrt{\Phi_{k}} W_{0}}{\sqrt{\Phi_{k}+W_{0}}}, \\
& \left|\int_{T}^{t}\left([W(\theta-T)+J(\theta)] e^{-(R / L)(\theta-T)} /(2 \sqrt{L})\right) \mathrm{d} \theta\right| \leq \frac{c_{k} \sqrt{\Phi_{k}} W_{0}}{\sqrt{\Phi_{k}+W_{0}}}
\end{aligned}
$$

or in view of the inequality

$$
\begin{aligned}
& \left|\int_{T}^{t}\left([W(\theta-T)+J(\theta)] e^{-(R / L)(\theta-T)} /(2 \sqrt{L})\right) \mathrm{d} \theta\right| \\
\leq & \left(\left(W_{0}+J_{0} e^{-\mu T}\right) /(2 \sqrt{L})\right)\left(\left(e^{(\mu-(R / L)) T_{0}}-1\right) /(\mu-(R / L))\right)
\end{aligned}
$$

we obtain

$$
\frac{W_{0}+J_{0} e^{-\mu T}}{2 \sqrt{L}} \frac{e^{(\mu-(R / L)) T_{0}}-1}{\mu-(R / L)} \leq \frac{c_{k} \sqrt{\Phi_{k}} W_{0}}{\sqrt{\Phi_{k}+W_{0}}}
$$

and

$$
\frac{W_{0} e^{-\mu T}+J_{0}}{2 \sqrt{L}} \frac{e^{(\mu-(R / L)) T_{0}}-1}{\mu-(R / L)} \leq \frac{c_{k} \sqrt{\Phi_{k}} W_{0}}{\sqrt{\Phi_{k}+W_{0}}} .
$$

For the $I-V$ characteristics we assume that they are polynomial functions

$$
R_{k}(i)=\sum_{n=1}^{m} r_{n}^{(k)} i^{n},(k=0,1) .
$$

Now we are able to formulate a periodic problem for the obtained neutral system:

$$
\begin{aligned}
& \frac{\mathrm{d} \bar{C}_{k}^{-1}(I)}{\mathrm{d} I}=\left\{\begin{array}{l}
\frac{1}{c_{k}^{2} \Phi_{k}}\left(-I-\frac{I^{2}+2 c_{k}^{2} \Phi_{k}^{2}}{\sqrt{I^{2}+4 c_{k}^{2} \Phi_{k}^{2}}}\right), I \in\left[\frac{-c_{k} \sqrt{\Phi_{k}} W_{0}}{\sqrt{\Phi_{k}+W_{0}}}, 0\right) \\
\frac{1}{c_{k}^{2} \Phi_{k}}\left(-I+\frac{I^{2}+2 c_{k}^{2} \Phi_{k}^{2}}{\sqrt{I^{2}+4 c_{k}^{2} \Phi_{k}^{2}}}\right), I \in\left(0, \frac{c_{k} \sqrt{\Phi_{k}} W_{0}}{\sqrt{\Phi_{k}-W_{0}}}\right],
\end{array}\right. \\
& \frac{\mathrm{d} W(t)}{\mathrm{d} t}=-\frac{\mathrm{d} J(t-T)}{\mathrm{d} t}+\frac{R}{L} W(t)+\frac{R}{L} J(t-T)+\left(2 e^{(R / L)(t-T)} \sqrt{L} E_{0}(t-T)-(W(t)-J(t-T)) Z_{0}\right) / \tilde{L}_{0} \\
& -\frac{2 e^{(R / L)(t-T)} \sqrt{L}}{\tilde{L}_{0}} R_{0}\left(\frac{e^{-(R / L)(t-T)}}{2 \sqrt{L}}(W(t)+J(t-T))\right) \\
& -\frac{2 e^{(R / L)(t-T)} \sqrt{L}}{\tilde{L}_{0}} \bar{C}_{0}^{-1}\left(\int_{T}^{t} \frac{e^{-(R / L)(t-T)}}{2 \sqrt{L}}(W(\theta)+J(\theta-T)) \mathrm{d} \theta\right) \equiv U(W, J)(t) . \\
& \frac{\mathrm{d} J(t)}{\mathrm{d} t}=-\frac{\mathrm{d} W(t-T)}{\mathrm{d} t}+\frac{R}{L} W(t-T)+\frac{R}{L} J(t)-2 e^{(R / L)(t-T)} \sqrt{L} E_{1}(t-T) / \tilde{L}_{1} \\
& +\left((W(t-T)-J(t)) Z_{0}\right) / \tilde{L}_{1}-\frac{2 e^{(R / L)(t-T)} \sqrt{L}}{\tilde{L}_{1}} R_{1} \frac{e^{-(R / L)(t-T)}}{2 \sqrt{L}}(W(t-T)+J(t)) \\
& -\frac{2 e^{-(R / L)(t-T)} \sqrt{L}}{\tilde{L}_{1}} \bar{C}_{1}^{-1}\left(\int_{T}^{t} \frac{e^{-(R / L)(t-T)}}{2 \sqrt{L}}(W(\theta-T)+J(\theta)) \mathrm{d} \theta\right) \equiv I(W, J)(s), t \in\left[T, T+T_{0}\right],
\end{aligned}
$$




$$
\begin{aligned}
& W(t)=v_{0}(t), \dot{W}(t)=\dot{v}_{0}(t), \\
& J(t)=v_{0}(t), \dot{J}(t)=i_{0}(t), \quad t \in[0, T] .
\end{aligned}
$$

The initial functions $v_{0}(t), \iota_{0}(t)$ can be obtained from the initial conditions (7) by shifting along the characteristics of the initial functions $u_{0}(x), i_{0}(x)$ (cf. [9-11]).

\section{Main Results}

Here we formulate conditions for the existence-uniqueness of a periodic solution of neutral functional differential system (27), (28) (for definition of neutral equation see [15]). First we define a suitable operator generating by the right-hand sides of the Equations (27), (28). We find a $T_{0}$-periodic solution of Equations (27), (28) on the interval $\left[T, T+T_{0}\right)$ coinciding with prescribed $T_{0}$ - periodic function on $[0, T]$ and then one can continue it periodically because our system is autonomous one.

By $L_{T_{0}}^{1, \infty}[T, \infty)$ we mean the space consisting of all measurable essentially bounded $T_{0}$ - periodic functions whose derivatives are also essentially bounded and $T_{0}-$ periodic.

Introduce the sets:

$$
\begin{aligned}
M_{u}= & \left\{f \in L_{T_{0}}^{1, \infty}[T, \infty): \int_{T_{0}}^{T+T_{0}} f(t) \mathrm{d} t=0 \wedge f(t)=v_{0}(t),\right. \\
& t \in[-T, T]\}, \\
M_{i}= & \left\{f \in L_{T_{0}}^{1, \infty}[T, \infty): \int_{T}^{T+T_{0}} f(t) \mathrm{d} t=0 \wedge f(t)=t_{0}(t),\right. \\
& t \in[-T, T]\} .
\end{aligned}
$$

Lemma 1. If $f(.) \in M_{u}$ (respectively $f(.) \in M_{i}$ ) then $F(t)=\int_{T}^{t} f(\tau) \mathrm{d} \tau$ is $T_{0}$ - periodic function.

Assumption (E) $E_{k}(.) \in L_{T_{0}}^{1, \infty}[-T, \infty), \int_{T}^{T+T_{0}} E_{k}(t) \mathrm{d} t=0$, $\left|E_{k}(t)\right| \leq W_{0} e^{\mu(t-T)}, t \in\left[T, T+T_{0}\right], E_{k}(-T)=0(k=0,1)$.

Assumption (IN) $v_{0}(t), \iota_{0}(t)$ are essentially bounded $T_{0}$ - periodic functions where $T=m T_{0}, m \in\{2,3,4,5, \cdots\}$, $v_{0}(0)=l_{0}(0)=0, \quad\left|v_{0} t\right| \leq W_{0},\left|l_{0}(t)\right| \leq J_{0}$. Here $W_{0}, J_{0}$ are prescribed positive constants.

Lemma 2. If the assumptions (IN) and (E) are fulfilled and $(W, J) \in M_{u} \times M_{i}$ then $R_{0}\left(\Pi_{0}(W, J)(t)\right)$,

$$
R_{1}\left(\Pi_{1}(W, J)(t)\right), \quad \Psi_{0}(t)=\int_{T}^{t}\left(\Pi_{0}(W, J)(\tau)\right) \mathrm{d} \tau
$$

and $\Psi_{1}(t)=\int_{T}^{t}\left(\Pi_{1}(W, J)(\tau)\right) \mathrm{d} \tau$ are $T_{0}-$ periodic func-
tions.

Define the operator $B=\left(B_{u}, B_{i}\right)$ on the set $M_{u} \times M_{i}$ by the formulas:

$$
\begin{aligned}
& B_{u}(W, J)(t):=v_{0}(t), t \in[0, T], \\
& B_{i}(W, J)(t):=l_{0}(t), t \in[0, T], \\
& B_{u}(W, J)(t):= \int_{T}^{t} U(W, J)(s) \mathrm{d} s \\
&-\left(\frac{t-T}{T_{0}}-\frac{1}{2}\right) \int_{T}^{T+T_{0}} U(W, J)(s) \mathrm{d} s \\
&-\frac{1}{T_{0}} \int_{T}^{T+T_{0}} \int_{T}^{t} U(W, J)(s) \mathrm{d} s \mathrm{~d} t, t \in\left[T, T+T_{0}\right] \\
& B_{i}(W, J)(t):= \int_{T}^{t} I(W, J)(s) \mathrm{d} s \\
&-\left(\frac{t-T}{T_{0}}-\frac{1}{2}\right)^{T} \int_{T}^{T+T_{0}} I(W, J)(s) \mathrm{d} s \\
&-\frac{1}{T_{0}} \int_{T}^{T+T_{0}} \int_{T}^{t} I(W, J)(s) \mathrm{d} s \mathrm{~d} t, t \in\left[T, T+T_{0}\right] .
\end{aligned}
$$

Remark 1. It is easy to see that changing the integration order one obtains

$$
\begin{aligned}
\int_{T}^{T+T_{0}} \int_{T}^{t} U(W, J)(s) \mathrm{d} s \mathrm{~d} t= & \left(T+T_{0}\right) \int_{T}^{T+T_{0}} U(W, J)(s) \mathrm{d} s \\
& -\int_{T}^{T+T_{0}} s U(W, J)(s) \mathrm{d} s
\end{aligned}
$$

and

$$
\begin{aligned}
\int_{T}^{T+T_{0}} \int_{T}^{t} I(W, J)(s) \mathrm{d} s \mathrm{~d} t= & \left(T+T_{0}\right) \int_{T}^{T+T_{0}} I(W, J)(s) \mathrm{d} s \\
& -\int_{T}^{T+T_{0}} s I(W, J)(s) \mathrm{d} s .
\end{aligned}
$$

Lemma 3. The periodic problem (27), (28) has a solution $(W(),. J().) \in M_{u} \times M_{i}$ iff the operator $B$ has a fixed point $(W, J) \in M_{u} \times M_{i}$, that is, $W=B_{u}(W, J)$, $J=B_{i}(W, J)$.

Introduce the sets

$$
\begin{gathered}
X_{u}=\left\{f \in M_{u}:|f(t)| \leq W_{0} e^{\mu(t-T)}, t \in\left[T, T+T_{0}\right]\right\}, \\
X_{i}=\left\{f \in M_{i}:|f(t)| \leq J_{0} e^{\mu(t-T)}, t \in\left[T, T+T_{0}\right]\right\} .
\end{gathered}
$$

Here the constant $\mu>0$ will be prescribed below.

The sets $X_{u}$ and $X_{i}$ turn out into metric spaces with metrics

$$
\begin{aligned}
& \rho(W, \bar{W})=\operatorname{ess} \sup \left\{e^{-\mu(t-T)}|W(t)-\bar{W}(t)|: t \in\left[T, T+T_{0}\right]\right\}, \\
& \rho(\dot{W}, \dot{\bar{W}})=\operatorname{ess} \sup \left\{e^{-\mu(t-T)}|\dot{W}(t)-\dot{\bar{W}}(t)|: t \in\left[T, T+T_{0}\right]\right\}, \\
& \rho(J, \bar{J})=\operatorname{ess} \sup \left\{e^{-\mu(t-T)}|J(t)-\bar{J}(t)|: t \in\left[T, T+T_{0}\right]\right\},
\end{aligned}
$$


$\rho(\dot{J}, \dot{\bar{J}})=\operatorname{ess} \sup \left\{e^{-\mu(t-T)}|\dot{J}(t)-\dot{\bar{J}}(t)|: t \in\left[T, T+T_{0}\right]\right\}$.

Define a metric on $X_{u} \times X_{i}$ in the following way:

$$
\begin{aligned}
& \rho((W, J),(\bar{W}, \bar{J})) \\
= & \max \{\rho(W, \bar{W}), \rho(J, \bar{J}), \rho(\dot{W}, \dot{\bar{W}}), \rho(\dot{J}, \dot{\bar{J}})\} .
\end{aligned}
$$

Assumption (LC): $\frac{e^{(\mu-(R / L)) T_{0}}\left(W_{0}+J_{0} e^{-\mu T}\right)}{2 \sqrt{L}} \leq i_{0}$,

$$
\frac{e^{(\mu-(R / L)) T_{0}}\left(W_{0} e^{-\mu T}+J_{0}\right)}{2 \sqrt{L}} \leq i_{0}
$$$$
\frac{W_{0}+J_{0} e^{-\mu T}}{2 \sqrt{L}} \frac{e^{(\mu-R / L) T_{0}}-1}{\mu-(R / L)} \leq \frac{c_{k} \sqrt{\Phi_{k}} W_{0}}{\sqrt{\Phi_{k}+W_{0}}},
$$$$
\left|B_{u}(W, J)(t) \leq\right| \int_{T}^{t} U(W, J)(s) \mathrm{d} s\left|+\frac{1}{2}\right| \int_{T}^{T+T_{0}} U(W, J)(s) \mathrm{d} s \mid
$$$$
+\frac{1}{T_{0}} \int_{T}^{T+T_{0}}\left|\int_{T}^{t} U(W, J)(s) \mathrm{d} s\right| \mathrm{d} t \leq e^{\mu(t-T)}\left[\frac{e^{\mu_{0}}+\mu_{0}-1}{\mu_{0}} e^{-\mu T} J_{0}\right.
$$$$
+\frac{\left(\mu_{0}+2\right) e^{\mu_{0}}+\mu_{0}-2}{2 \mu_{0}}\left(\frac{R}{L}+\frac{Z_{0}}{\bar{L}_{0}}\right) \frac{\left(W_{0}+J_{0} e^{-\mu T}\right)}{\mu}+\frac{2 \sqrt{L} W_{0}\left(e^{-\mu T}+1\right)}{\bar{L}_{0}(\mu+R / L)} \mathrm{e}^{(R / L) T_{0}} \frac{\left(\mu_{0}+2\right) e^{\mu_{0}}+2 \mu_{0}-2}{\mu_{0}}
$$$$
\left.+\frac{1}{\bar{L}_{0}} \sum_{n=1}^{m} \frac{\left|r_{n}^{(0)}\right|\left(W_{0}+J_{0} e^{-\mu T}\right)^{n}}{n(2 \sqrt{L})^{n-1}(\mu-(((n-1) R) /(n R)))} \times\left(e^{(n-1)(\mu-(R / L)) T_{0}} \frac{2 e^{\mu_{0}}+3 \mu_{0}-2}{2 \mu_{0}}-\frac{1}{2}\right)\right] \leq e^{\mu(t-T)} W_{0} .
$$

Using inequality (24) and proceeding as in [11] we obtain (for sufficiently large $\mu>(R / L)$ ):

For the second component of the operator we obtain

$$
\begin{aligned}
\mid B_{i}(W, J)(t) \leq & \leq\left|\int_{T}^{t} I(W, J)(s) \mathrm{d} s\right|+\frac{1}{2}\left|\int_{T}^{T+T_{0}} I(W, J)(s) \mathrm{d} s\right|+\frac{1}{T_{0}} \int_{T}^{T+T_{0}}\left|\int_{T}^{t} I(W, J)(s) \mathrm{d} s\right| \mathrm{d} t \\
\leq & e^{\mu(t-T)}\left[W_{0} e^{-\mu T}\left(e^{\mu_{0}}+\mu_{0}-1\right) / \mu_{0}+\left(W_{0}\left(e^{-\mu T}+1\right) / \mu\right)\right. \\
& \times\left((R / L)+\left(Z_{0} / \bar{L}_{1}\right)\right)\left(\left(\mu_{0}+2\right) e^{\mu_{0}}+\mu_{0}-2\right) /\left(2 \mu_{0}\right)+\left(4 e^{(R / L) T_{0}}+e^{(\mu+(R / L)) T_{0}}-1\right) \frac{\sqrt{L} W_{0}\left(e^{-\mu T}+1\right)}{\bar{L}_{1}((R / L)+\mu)} \\
& \left.+\left(2 e^{\mu_{0}}+3 \mu_{0}-2\right) /\left(2 \mu_{0} \bar{L}_{1}\right) \times \sum_{n=1}^{m} \frac{\left|r_{n}^{(1)}\right|\left(W_{0} e^{-\mu T}+J_{0}\right)^{n}}{(2 \sqrt{L})^{n-1} n} \frac{e^{(n \mu-(n-1)(R / L)) T_{0}}-1}{\mu-(((n-1) R) /(n R))}\right] \leq J_{0} e^{\mu(t-T)} .
\end{aligned}
$$

Therefore the operator $B$ maps the set $X_{u} \times X_{i}$ into itself. In what follows we show that $B$ is contractive operator. We need the following preliminary inequalities

$$
\begin{aligned}
& \left|\Pi_{0}(W, J)(t)-\Pi_{0}(\bar{W}, \bar{J})(t)\right| \leq \frac{e^{(\mu-(R / L))(t-T)}}{2 \mu \sqrt{L}}\left(\rho(\dot{W}, \dot{\bar{W}})+\rho(\dot{J}, \dot{\bar{J}}) e^{-\mu T}\right), \\
& \left|\Pi_{1}(W, J)(t)-\Pi_{1}(\bar{W}, \bar{J})(t)\right| \leq \frac{e^{(\mu-(R / L))(t-T)}}{2 \mu \sqrt{L}}\left(\rho(\dot{W}, \dot{\bar{W}}) e^{-\mu T}+\rho(\dot{J}, \dot{\bar{J}})\right),
\end{aligned}
$$




$$
\begin{aligned}
& \left|\left[\Pi_{0}(W, J)(t)\right]^{n}-\left[\Pi_{0}(\bar{W}, \bar{J})(t)\right]^{n}\right| \\
\leq & n\left[e^{(\mu-(R / L)) T_{0}}\left(W_{0}+J_{0} e^{-\mu T}\right) /(2 \sqrt{L})\right]^{n-1} \times e^{(\mu-(R / L))(t-T)}\left(\rho(\dot{W}, \dot{\bar{W}})+\rho(\dot{J}, \dot{\bar{J}}) e^{-\mu T}\right) /(2 \mu \sqrt{L}) . \\
& \left|\left[\Pi_{1}(W, J)(t)\right]^{n}-\left[\Pi_{1}(\bar{W}, \bar{J})(t)\right]^{n}\right| \\
\leq & n\left[e^{(\mu-(R / L)) T_{0}}\left(W_{0} e^{-\mu T}+J_{0}\right) /(2 \sqrt{L})\right]^{n-1} \times e^{(\mu-(R / L))(t-T)}\left(\rho(\dot{W}, \dot{\bar{W}}) e^{-\mu T}+\rho(\dot{J}, \dot{\bar{J}})\right) /(2 \mu \sqrt{L}) .
\end{aligned}
$$

Then for the first operator component we obtain:

$$
\begin{aligned}
& \left|B_{u}(W, J)(t)-B_{u}(\bar{W}, \bar{J})(t)\right| \\
\leq & \int_{T}^{t}|U(W, J)(s)-U(\bar{W}, \bar{J})(s)| \mathrm{d} s+\left(\frac{t-T}{T_{0}}-\frac{1}{2}\right)\left|\int_{T}^{T+T_{0}} U(W, J)(s) \mathrm{d} s-\int_{T}^{T+T_{0}} U(\bar{W}, \bar{J})(s) \mathrm{d} s\right| \\
& +\frac{1}{T_{0}} \int_{T}^{T+T_{0}}\left|\int_{T}^{t} U(W, J)(s) \mathrm{d} s-\int_{T}^{t} U(\bar{W}, \bar{J})(s) \mathrm{d} s\right| \mathrm{d} t \\
\leq & e^{\mu(t-T)}[\rho(W, J),(\bar{W}, \bar{J})) \frac{e^{\mu_{0}}+\mu_{0}-1}{\mu_{0}} \frac{e^{-\mu T}}{\mu}+\frac{\left(\mu_{0}+2\right) e^{\mu_{0}}+\mu_{0}-2}{2 \mu_{0}} \frac{1+e^{-\mu T}}{\mu^{2}} \\
& \left.\cdot\left((R / L)+\left(Z_{0} / \bar{L}_{0}\right)+\frac{1}{\bar{L}} \sum_{n=1}^{m}\left|r_{n}^{(0)}\right| \frac{n e^{(n-1)(\mu-(R / L)) T_{0}}\left(W_{0}+J_{0} e^{-\mu T}\right)^{n-1}}{(2 \sqrt{L})^{n-1}}+2 \sqrt{1+\left(W_{0} / \Phi_{0}\right)} /\left(c_{0} \bar{L}_{0}(\mu-(R / L))\right)\right)\right] \\
\equiv & e^{\mu(t-T)} K_{u} \rho((W, J),(\bar{W}, \bar{J})) .
\end{aligned}
$$

Further on we have

$$
\begin{aligned}
& \left|B_{i}(W, J)(t)-B_{i}(\bar{W}, \bar{J})(t)\right| \\
\leq & \int_{T}^{t}|I(W, J)(s)-I(\bar{W}, \bar{J})(s)| \mathrm{d} s+\left(\frac{t-T}{T_{0}}-\frac{1}{2}\right)\left|\int_{T}^{T+T_{0}} I(W, J)(s) \mathrm{d} s-\int_{T}^{T+T_{0}} I(\bar{W}, \bar{J})(s) \mathrm{d} s\right| \\
& +\frac{1}{T_{0}} \int_{T}^{T+T_{0}}\left|\int_{T}^{t} I(W, J)(s) \mathrm{d} s-\int_{T}^{t} I(W, \bar{J})(s) \mathrm{d} s\right| \mathrm{d} t \\
\leq & e^{\mu(t-T)} \rho((W, J),(\bar{J}, \bar{W}))\left[\frac{e^{\mu_{0}}+\mu_{0}-1}{\mu_{0}} \frac{e^{-\mu T}}{\mu}+\frac{\left(\mu_{0}+2\right) e^{\mu_{0}}+\mu_{0}-2}{2 \mu_{0}} \frac{\left(e^{-\mu T}+1\right)}{\mu^{2}}\right. \\
& \cdot\left(\left((R / L)+\left(Z_{0} / \overline{L_{0}}\right)+\frac{2}{\bar{L}_{1}} \sum_{n=1}^{m}\left|r_{n}^{(1)}\right| \frac{n e^{(n-1)(\mu-(R / L)) T_{0}}\left(W_{0} e^{-\mu T}+J_{0}\right)^{n-1}}{(2 \sqrt{L})^{n-1}}+\frac{2}{c_{1} \bar{L}_{1}(\mu-(R / L))} \sqrt{1+\left(W_{0} / \Phi_{1}\right)}\right)\right] \\
\equiv & e^{\mu(t-T)} K_{i} \rho((W, J),(\bar{J}, \bar{W})) .
\end{aligned}
$$

Finally we have to obtain an estimate for $t \in\left[T, T+T_{0}\right]$. It is easy to prove the following inequalities: 


$$
\begin{aligned}
& \quad\left|\dot{B}_{u}(W, J)(t)-\dot{B}_{u}(\bar{W}, \bar{J})(t)\right| \\
& \leq|U(W, J)(t)-U(\bar{W}, \bar{J})(t)|+\frac{1}{T_{0}} \int_{T}^{T+T_{0}} U(W, J)(s) \mathrm{d} s-\int_{T}^{T+T_{0}} U(\bar{W}, \bar{J})(s) \mathrm{d} s \mid \\
& \leq e^{\mu(t-T)} \rho((W, J),(\bar{W}, \bar{J}))\left[e^{-\mu T}+\frac{e^{\mu_{0}}+\mu_{0}-1}{\mu_{0}} \frac{\left(1+e^{-\mu T}\right)}{\mu} \times\left(\frac{R}{L}+\frac{Z_{0}}{\bar{L}_{0}}+\frac{1}{\bar{L}_{0}} \sum_{n=1}^{m}\left|r_{n}^{(0)}\right| \frac{n e^{(n-1)(\mu-(R / L)) T_{0}}\left(W_{0}+J_{0} e^{-\mu T}\right)^{n-1}}{(2 \sqrt{L})^{n-1}}\right.\right. \\
& \left.\left.\quad+\frac{2 \sqrt{1+W_{0} / \Phi_{0}}}{\bar{L}_{0} C_{0}(\mu-(R / L))}\right)\right] \\
& \equiv e^{\mu(t-T)} \dot{K}_{u} \rho((W, J),(\bar{J}, \bar{W})) .
\end{aligned}
$$

For the derivative of the second component of $B$ we obtain

$$
\begin{aligned}
& \left|\dot{B}_{i}(W, J)(t)-\dot{B}_{i}(\bar{W}, \bar{J})(t)\right| \\
\leq & |I(W, J)(t)-I(\bar{W}, \bar{J})(t)|+\frac{1}{T_{0}}\left|\int_{T}^{T+T_{0}} I(W, J)(s) \mathrm{d} s-\int_{T}^{T+T_{0}} I(\bar{W}, \bar{J})(s) \mathrm{d} s\right| \\
\leq & e^{\mu(t-T)} \rho((W, J),(\bar{W}, \bar{J}))\left[e^{-\mu T}+\frac{e^{\mu_{0}}+\mu_{0}-1}{\mu_{0}} \frac{\left(e^{-\mu T}+1\right)}{\mu}\right. \\
& \left.\times\left(\frac{R}{L}+\frac{Z_{0}}{\bar{L}_{1}}+\frac{1}{\bar{L}_{1}} \sum_{n=1}^{m} n\left|r_{n}^{(1)}\right| \frac{e^{(n-1)\left(\mu-\frac{R}{L}\right)^{T_{0}}}\left(W_{0} e^{-\mu T}+J_{0}\right)^{n-1}}{(2 \sqrt{L})^{n-1}}+\frac{2 \sqrt{1+W_{0} / \Phi_{1}}}{c_{1} \bar{L}_{1}(\mu-(R / L))}\right)\right] \\
\equiv & e^{\mu(t-T)} \dot{K}_{i} \rho((W, J),(\bar{J}, \bar{W})) .
\end{aligned}
$$

The above inequalities imply

$$
\rho(B(W, J), B(\bar{W}, \bar{J})) \leq K \rho((W, J),(\bar{W}, \bar{J}))
$$

where $K=\max \left\{K_{u}, K_{i}, \dot{K}_{u}, \dot{K}_{i}\right\}$ should be chosen smaller than 1.

Therefore $B$ is contractive operator and has a unique fixed point in $M$ (cf. [18]). It is a unique periodic solution of system (26), (27).

Theorem 1 is thus proved.

\section{Numerical Example}

For a transmission line with length $\Lambda=1 \mathrm{~m}$,

$L=0.45 \mu \mathrm{H} / \mathrm{m}, C=80 \mathrm{pF} / \mathrm{m}$, cross-section area

$S=6 \mathrm{~mm}^{2}$, specific resistance for the cuprum

$\rho_{c}=0.0175$, that is, $R=\left(\rho_{c} \Lambda\right) / S \approx 3.10^{-3} \Omega$,

$v=1 / \sqrt{L C}=1 /\left(6 \cdot 10^{-9}\right)=1.66 \cdot 10^{8}$,

$Z_{0}=\sqrt{L / C}=75 \Omega$. Then $T=\Lambda \sqrt{L C}=6 \cdot 10^{-9} \mathrm{sec}$.

Let us check the propagation of millimeter waves $\lambda_{0}=(1 / 6) \cdot 10^{-3} \mathrm{~m}$. We have

$$
f_{0}=1 /\left(\lambda_{0} \sqrt{L C}\right)=10^{12} \mathrm{~Hz} \Rightarrow T_{0}=1 / f_{0}=10^{-12} .
$$

We choose $\mu=2 \cdot 10^{12}$. Then $\mu T_{0}=\mu_{0}=2$ and $T=6 \cdot 10^{-9} \cdot 2 \cdot 10^{12} T_{0}=12000 T_{0}$. Consequently

$$
\begin{aligned}
& \mu T=2 \cdot 10^{12} \cdot 6 \cdot 10^{-9} \approx 12 \cdot 10^{3} \Rightarrow e^{-\mu T} \approx 0, R T / L \approx 0, \\
& R / L=0.0029 /\left(0.45 \cdot 10^{-6}\right) \approx 0.6 \cdot 10^{4}, e^{ \pm(R T / L)} \approx e^{0} \approx 1 \\
& \mu \pm(R / L)=2 \cdot 10^{12} \pm 10^{4}=10^{12}\left(2 \pm 10^{-8}\right) \approx 2 \cdot 10^{12}, \\
& (R / L) T_{0} \approx 0 \Rightarrow e^{(R / L) T_{0}}-1 \approx 6.6 \cdot 10^{-6},
\end{aligned}
$$$$
\mu-(R / L) \approx 10^{12}, e^{(\mu-(R / L)) T_{0}}=e^{\mu_{0}} e^{-(R / L) T_{0}} \approx e^{2} .
$$

We choose resistive elements with the following $V-I$ characteristics $R_{0}(u)=R_{1}(u)=0.028 u-0.125 u^{3}$ i.e. $r_{1}=0.028, r_{2}=0, r_{3}=0.125$, and inductive element with $L_{0}(i)=L_{1}(i)=3 i-(1 / 12) i^{3}$. Then

$$
\tilde{L}_{0}(i)=i\left(\mathrm{~d} L_{0}(i) / \mathrm{d} i\right)+L_{0}(i)=6 i-(1 / 3) i^{3} .
$$

If we choose $i_{0}=1$ one obtains

$$
6 i-(1 / 3) i^{3}>6-(1 / 3)=17 / 3
$$

and consequently $1 / \bar{L}=3 / 17$.

Let us take

$$
C_{0}(u)=C_{1}(u)=c / \sqrt{1-(u / \Phi)}=c \sqrt{\Phi} / \sqrt{\Phi-u},
$$

where $h=2, c=50 p F=5 \cdot 10^{-11} F$ and $\Phi=0.4 \mathrm{~V} \Rightarrow$ 
$W_{0}<0.4$. Then the inequalities of Theorem 1 become

$$
\begin{aligned}
& e^{(\mu-(R / L)) T_{0}}\left(W_{0}+J_{0} e^{-\mu T}\right) /(2 \sqrt{L}) \leq 1 \\
& \Rightarrow e^{2} W_{0} /(2 \sqrt{L}) \leq 1 \Rightarrow W_{0} \leq 2 \sqrt{L} / e^{2} \approx 0.18 \cdot 10^{-3}, \\
& e^{(\mu-R / L) T_{0}}\left(W_{0} e^{-\mu T}+J_{0}\right) /(2 \sqrt{L}) \leq 1 \\
& \Rightarrow e^{2} J_{0} /(2 \sqrt{L}) \leq 1 \Rightarrow J_{0} \leq 2 \sqrt{L} / e^{2} \approx 0.18 \cdot 10^{-3}
\end{aligned}
$$

$$
\begin{gathered}
\frac{W_{0}}{2 \sqrt{L}} \frac{e^{2}-1}{\mu} \leq \frac{c W_{0} \sqrt{\Phi}}{\sqrt{\Phi+W_{0}}} \Leftrightarrow \sqrt{\Phi+W_{0}} \leq \frac{2 c \mu \sqrt{L \Phi}}{e^{2}-1} \\
\Rightarrow W_{0} \leq \min \left\{13.25^{2}-0.4 ; 0.18 \cdot 10^{-3}\right\}=0.18 \cdot 10^{-3}, \\
\frac{J_{0}}{2 \sqrt{L}} \frac{e^{2}-1}{\mu} \leq \frac{c W_{0} \sqrt{\Phi}}{\sqrt{\Phi+W_{0}}} \\
\Rightarrow J_{0} \leq \frac{2 \cdot c \sqrt{L} \sqrt{\Phi} \cdot W_{0} \mu}{\left(e^{2}-1\right) \sqrt{\Phi+W_{0}}} \approx 6 \cdot 10^{-6} ;
\end{gathered}
$$

and

$$
\begin{aligned}
& \frac{1}{\mu}\left[e^{2}\left(\frac{R}{L}+\frac{Z_{0}}{\bar{L}}\right)+\frac{\left(4 e^{2}+2\right) \sqrt{L}}{\bar{L}}+\frac{1}{\bar{L}}\left(0,028 \frac{e^{2}+1}{2}+\frac{0,125 W_{0}^{2}}{L} \frac{e^{6}+2 e^{4}-1}{24}\right)\right] \leq 1, \\
& \frac{1}{\mu}\left[\left(\frac{R}{L}+\frac{Z_{0}}{\bar{L}}\right) e^{2} W_{0}+\frac{\left(e^{2}+3\right) \sqrt{L} W_{0}}{\bar{L}}+\frac{e^{2}+2}{2 \bar{L}}\left(0.028 J_{0}\left(e^{2}-1\right)+\frac{0,125 J_{0}^{3}}{12 L}\left(e^{6}-1\right)\right)\right] \leq J_{0}, \\
& \dot{K}_{u}=\frac{e^{2}+1}{2 \mu}\left[\frac{R}{L}+\frac{Z_{0}}{\bar{L}}+\frac{1}{\bar{L}}\left(0.028+0.125 \frac{3 e^{4} W_{0}^{2}}{4 L}\right)+2 \sqrt{1+\left(W_{0} / \Phi\right)} /(\mu \bar{L} c)\right]<1, \\
& \dot{K}_{i}=\frac{e^{2}+1}{2 \mu}\left[\frac{R}{L}+\frac{Z_{0}}{\bar{L}}+\frac{1}{\bar{L}}\left(0.028+0.125 \frac{3 e^{4} J_{0}^{2}}{4 L}\right)+2 \sqrt{1+\left(W_{0} / \Phi\right)} /(\mu \bar{L} c)\right]<1 .
\end{aligned}
$$

Taking into account $R / L+Z_{0} / \bar{L}=10^{4}\left(0.6+13.24 \cdot 10^{-4}\right) \approx 0.6 \cdot 10^{4}, \quad W_{0}<0.18 \cdot 10^{-3}$ and $J_{0} \leq 3 \cdot 10^{-6}$ we see that

$$
\begin{aligned}
& 1 /\left(2 \cdot 10^{12}\right)\left[4.44 \cdot 10^{4}+5.58 \sqrt{0.45 \cdot 10^{-6}}+(13 / 17)(0.118+3.4)\right] \leq 1, \\
& 10^{-6}\left[8+0.83\left(0.54 \cdot 10^{-6}+\left(3.375 \cdot 10^{-12}\right) 404 / 5.4\right)\right] \leq 6, \\
& K_{u}, K_{i}<\dot{K}_{i} \leq \dot{K}_{u} \approx \approx\left(2,1 / 10^{12}\right)\left(0.6 \cdot 10^{4}+0.072+0.0036\right) 13 \cdot 10^{-7}<1 .
\end{aligned}
$$

Most often the initial approximation is chosen to be simple functions, namely:

$$
\begin{aligned}
& W^{(0)}(t)=\left\{\begin{array}{c}
W_{0} \sin \omega_{0} t, t \in[0, T] \\
0, t \in\left[T, T+T_{0}\right]
\end{array} ;\right. \\
& J^{(0)}(t)=\left\{\begin{array}{c}
J_{0} \sin \omega_{0} t, t \in[0, T] \\
0, t \in\left[T, T+T_{0}\right]
\end{array}\right.
\end{aligned}
$$

$\left(\omega_{0}=2 \pi / T_{0}\right)$ and $E_{0}(t)=E_{1}(t)=E_{0} \sin \omega_{0} t$.

$$
\begin{aligned}
& -(1 / 3)\left(e^{-(R / L)(t-T)}(W(t)+J(t-T)) /(2 \sqrt{L})\right)^{3}, \\
& \tilde{L}_{1}(t-T) \\
= & 6\left(e^{-(R / L)(t-T)}(W(t-T)+J(t)) /(2 \sqrt{L})\right) \\
& -(1 / 3)\left(e^{-(R / L)(t-T)}(W(t-T)+J(t)) /(2 \sqrt{L})\right)^{3}
\end{aligned}
$$

Then we have $W^{(n+1)}(t)=B_{u}\left(W^{(n)}, J^{(n)}\right)$,

$$
J^{(n+1)}(t)=B_{i}\left(W^{(n)}, J^{(n)}\right)
$$

$(n=0,1,2, \cdots)$ and

$$
\begin{aligned}
& \rho\left(\left(W^{(n+1)}, J^{(n+1)}\right),\left(W^{(n)}, J^{(n)}\right)\right) \\
\leq & \left(1.13 \cdot 10^{-7}\right)^{n} /\left(1-1.13 \cdot 10^{-7}\right) \rho\left(\left(W^{(1)}, J^{(1)}\right),\left(W^{(0)}, J^{(0)}\right)\right), \\
& (n=1,2 \cdots)
\end{aligned}
$$

We notice that $\tilde{L}_{0}(i)=\tilde{L}_{1}(i)=6 i-(1 / 3) i^{3}$ imply

$$
\begin{aligned}
& \tilde{L}_{0}(t-T) \\
= & 6\left(e^{-(R / L)(t-T)}(W(t)+J(t-T)) /(2 \sqrt{L})\right)
\end{aligned}
$$

and therefore (recall $\left|\Pi_{0}\right| \leq i_{0}=1,\left|\Pi_{1}\right| \leq i_{0}=1$ ):

$$
\begin{aligned}
& \quad\left|2 \sqrt{L} \int_{T}^{t} e^{(R / L)(s-T)} R_{0}\left(\Pi_{0}\left(W^{(0)}, J^{(0)}\right)(s)\right) /\left(\tilde{L}_{0}(s-T)\right) \mathrm{d} s\right| \\
& \leq 2 \sqrt{L} \int_{T}^{t} e^{(R / L)(s-T)}\left|R_{0}\left(i_{0}\right)\right| /\left(\tilde{L}_{0}(s-T)\right) \mathrm{d} s \\
& \leq 2 \sqrt{L} \int_{T}^{t} e^{(R / L)(s-T)}(0,028+0,125) /(\bar{L}) \mathrm{d} s \\
& \leq 0.31 L \sqrt{L}\left(e^{(R / L) T_{0}}-1\right) /(\bar{L} R) \leq 0.074 \cdot 10^{-12} \approx 0 .
\end{aligned}
$$

Analogously 


$$
\left|2 \sqrt{L} \int_{T}^{t} e^{(R / L)(s-T)} R_{1}\left(\Pi_{1}\left(W^{(0)}, J^{(0)}\right)(s)\right) / \tilde{L}_{1}(s-T) \mathrm{d} s\right| \leq(6 \sqrt{L} / 17)(0.028+0.125)\left(e^{(R / L) T_{0}}-1\right) L / R \approx 0 .
$$

We find the first approximation

$$
\begin{aligned}
& W^{(1)}(t)=B_{u}\left(W^{(0)}, J^{(0)}\right)(t)=\int_{T}^{t} U\left(W^{(0)}, J^{(0)}\right)(s) \mathrm{d} s-\left(\frac{t-T}{T_{0}}-\frac{1}{2}\right) \int_{T}^{T+T_{0}} U\left(W^{(0)}, J^{(0)}\right)(s) \mathrm{d} s \\
- & \frac{1}{T_{0}} \int_{T}^{T+T_{0}} \int_{T}^{t} U\left(W^{(0)}, J^{(0)}\right)(s) \mathrm{d} s \mathrm{~d} t \\
= & -J_{0} \sin \omega_{0} t+(R / L) \int_{T}^{t} J_{0} \sin \omega_{0} s \mathrm{~d} s+2 E_{0} \sqrt{L} \int_{T}^{t} e^{(R / L)(s-T)} \sin \omega_{0} s /\left(\tilde{L}_{0}(s-T)\right) \mathrm{d} s \\
& +Z_{0} J_{0} \int_{T}^{t} \sin \omega_{0} s /\left(\tilde{L}_{0}(s-T)\right) \mathrm{d} s-2 \sqrt{L} \int_{T}^{t} \frac{e^{(R / L)(s-T)}}{\tilde{L}_{0}(s-T)} \frac{\bar{C}_{0}^{-1}}{2 \sqrt{L}}\left(\int_{T}^{s} e^{-(R / L)(\theta-T)} J_{0} \sin \omega_{0} \theta \mathrm{d} \theta\right) \mathrm{d} s \\
& -\left(\frac{t-T}{T_{0}}-\frac{1}{2}\right)\left[2 E_{0} \sqrt{L} \int_{T}^{T+T_{0}} e^{(R / L)(s-T)} \sin \omega_{0} s /\left(\tilde{L}_{0}(s-T)\right) \mathrm{d} s+Z_{0} J_{0} \int_{T}^{T+T_{0}} \sin \omega_{0} s /\left(\tilde{L}_{0}(s-T)\right) \mathrm{d} s\right. \\
& \left.-2 \sqrt{L} \int_{T}^{T+T_{0}} \frac{e^{(R / L)(s-T)}}{\tilde{L}_{0}(s-T)} \bar{C}_{0}^{-1}\left(\frac{J_{0}}{2 \sqrt{L}} \int_{T}^{s} e^{-(R / L)(\theta-T)} \sin \omega_{0} \theta \mathrm{d} \theta\right) \mathrm{d} s\right] \\
& -\frac{1}{T_{0}} \int_{T}^{T+T_{0}}\left[-J_{0} \sin \omega_{0} t+(R / L) \int_{T}^{t} J_{0} \sin \omega_{0} s \mathrm{~d} s+2 E_{0} \sqrt{L} \int_{T}^{t} e^{(R / L)(s-T)} \sin \omega_{0} s \tilde{L}_{0}(s-T) \mathrm{d} s\right. \\
& \left.+Z_{0} J_{0} \int_{T}^{t} \sin \omega_{0} s /\left(\tilde{L}_{0}(s-T)\right) \mathrm{d} s-2 \sqrt{L} \int_{T}^{t} \frac{e^{(R / L)(s-T)}}{\tilde{L}_{0}(s-T)} \bar{C}_{0}^{-1}\left(\frac{J_{0}}{2 \sqrt{L}} \int_{T}^{s} e^{-(R / L)(\theta-T)} \sin \omega_{0} \theta \mathrm{d} \theta\right) \mathrm{d} s\right] \mathrm{d} t .
\end{aligned}
$$

Since $\left|(R / L) \int_{T}^{t} J_{0} \sin \omega_{0} s \mathrm{~d} s\right| \approx J_{0} \cdot 10^{-9}$ and

$$
2 \sqrt{L}\left|\int_{T}^{t} \frac{e^{(R / L)(s-T)}}{\tilde{L}_{0}(s-T)} \bar{C}_{0}^{-1}\left(\frac{J_{0}}{2 \sqrt{L}} \int_{T}^{s} e^{-(R / L)(\theta-T)} \sin \omega_{0} \theta \mathrm{d} \theta\right) \mathrm{d} s\right| \leq W_{0} \frac{2 \sqrt{L}}{\bar{L}} \frac{L}{R}\left(e^{(R / L) T_{0}}-1\right) \approx W_{0} 3.6 \cdot 10^{-8} \approx 0
$$

we can disregard this terms and obtain

$$
\begin{aligned}
W^{(1)}(t)= & -J_{0} \sin \omega_{0} t+2 E_{0} \sqrt{L} \int_{T}^{t} \frac{e^{(R / L)(s-T)} \sin \omega_{0} s}{\tilde{L}_{0}(s-T)} \mathrm{d} s+Z_{0} J_{0} \int_{T}^{t} \sin \omega_{0} s /\left(\tilde{L}_{0}(s-T)\right) \mathrm{d} s \\
& -\left(\frac{t-T}{T_{0}}-\frac{1}{2}\right)\left(2 E_{0} \sqrt{L} \int_{T}^{T+T_{0}} e^{(R / L)(s-T)} \sin \omega_{0} s /\left(\tilde{L}_{0}(s-T)\right) \mathrm{d} s+Z_{0} J_{0} \int_{T}^{T+T_{0}} \sin \omega_{0} s /\left(\tilde{L}_{0}(s-T)\right) \mathrm{d} s\right) \\
& -\frac{1}{T_{0}} \int_{T}^{T+T_{0}}\left(2 E_{0} \sqrt{L} \int_{T}^{t} e^{(R / L)(s-T)} \sin \omega_{0} s /\left(\tilde{L}_{0}(s-T)\right) \mathrm{d} s+Z_{0} J_{0} \int_{T}^{t} \sin \omega_{0} s /\left(\tilde{L}_{0}(s-T)\right) \mathrm{d} s\right) \mathrm{d} t,
\end{aligned}
$$

and then

$$
\begin{aligned}
& \left|W^{(1)}(t)-W^{(0)}(t)\right| \\
\leq & J_{0}+\left(2 E_{0} L \sqrt{L} / R \bar{L}\right)\left(e^{(R / L) T_{0}}-1\right)+\left(Z_{0} J_{0} / \bar{L}\right)\left|\int_{T}^{t} e^{\mu(s-T)} \mathrm{d} s\right|+\left(E_{0} L \sqrt{L} / R \bar{L}\right)\left(e^{(R / L) T_{0}}-1\right) \\
& +\left(Z_{0} J_{0} / 2 \bar{L}\right)\left|\int_{T}^{T+T_{0}} e^{\mu(s-T)} \mathrm{d} s\right|+\frac{1}{T_{0}} \int_{T}^{T+T_{0}}\left(\left(2 E_{0} L \sqrt{L} / R \bar{L}\right)\left(e^{(R / L) T_{0}}-1\right)+\left(Z_{0} J_{0} / \bar{L}\right)\left|\int_{T}^{t} e^{\mu(s-T)} \mathrm{d} s\right|\right) \mathrm{d} t \leq J_{0} \\
& +\left(5 L^{3 / 2} / \bar{L} R\right)\left(e^{(R / L) T_{0}}-1\right) E_{0}+5 Z_{0} J_{0}\left(e^{\mu_{0}}-1\right) /(2 \bar{L} \mu) \\
\leq & e^{\mu_{0}} J_{0}=e^{2} J_{0} .
\end{aligned}
$$


For the derivative we have

$$
\begin{aligned}
\dot{W}^{(1)}(t)= & U\left(W^{(0)}, J^{(0)}\right)(t)-\frac{1}{T_{0}} \int_{T}^{T+T_{0}} U\left(W^{(0)}, J^{(0)}\right)(s) \mathrm{d} s \\
= & -\omega_{0} J_{0} \cos \omega_{0} t+\frac{R}{L} J_{0} \sin \omega_{0} t+\frac{2 e^{(R / L)(t-T)} E_{0} \sqrt{L}}{\tilde{L}_{0}(t-T)} \\
& +\frac{Z_{0}}{\tilde{L}_{0}} J_{0} \sin \omega_{0} t-\frac{2 e^{(R / L)(t-T)} \sqrt{L} R\left(\Pi_{0}\left(W^{(0)}, J^{(0)}\right)(t)\right)}{\tilde{L}_{0}(t-T)} \\
& -\frac{2 e^{(R / L)(t-T)} \sqrt{L}}{\tilde{L}_{0}(t-T)} \bar{C}_{0}^{-1}\left(\int_{T}^{t} \Pi_{0}\left(W^{(0)}, J^{(0)}\right)(\theta) \mathrm{d} \theta\right)-\frac{1}{T_{0}}\left(2 E \sqrt{L} \int_{T}^{T+T_{0}} e^{(R / L)(s-T) / \tilde{L}_{0}(s-T)} \mathrm{d} s\right) \\
& +\frac{1}{T_{0}}\left(Z_{0} \int_{T}^{T+T_{0}} J_{0} \sin \omega_{0} /\left(\tilde{L}_{0}(s-T)\right) \mathrm{d} s-2 \sqrt{L} \int_{T}^{T+T_{0}} e^{(R / L)(s-T)} R_{0}\left(\Pi_{0}\left(W^{(0)}, J^{(0)}\right)(s)\right) /\left(\tilde{L}_{0}(s-T)\right) \mathrm{d} s\right) \\
& +\frac{1}{T_{0}}\left(2 \sqrt{L} \int_{T}^{T+T_{0}} \frac{e^{(R / L)(s-T)}}{\tilde{L}_{0}(s-T)} \bar{C}_{0}^{-1}\left(\int_{T}^{s} \Pi_{0}\left(W^{(0)}, J^{(0)}\right)(\theta) \mathrm{d} \theta\right) \mathrm{d} s\right) .
\end{aligned}
$$

Then we obtain

$$
\begin{aligned}
& \left|\dot{W}^{(1)}(t)-\dot{W}^{(0)}(t)\right| \\
\leq & \left(\omega_{0}+\frac{R}{L}+\frac{Z_{0}}{\bar{L}}+\frac{Z_{0}}{\bar{L}}\left(e^{\mu_{0}}-1\right) / \mu_{0}\right) J_{0}+(2 \sqrt{L} / \bar{L})\left(e^{R T_{0} / L}+\left(e^{R T_{0} / L}-1\right) /\left(R T_{0} / L\right)\right)\left(E_{0}+W_{0}\right) \\
& +(2 \sqrt{L} / \bar{L})(0.028+0.125)\left(e^{R T_{0} / L}+\left(e^{R T_{0} / L}-1\right) /\left(R T_{0} / L\right)\right)+(2 \sqrt{L} / \bar{L})\left(e^{R T_{0} / L}-1\right) /\left(R T_{0} / L\right) W_{0} .
\end{aligned}
$$

Since $\left(e^{R T_{0} / L}-1\right) /\left(R T_{0} / L\right) \approx 1, E_{0}=0.5 V$ we have

$$
\left|\dot{W}^{(1)}(t)-\dot{W}^{(0)}(t)\right| \leq\left(2 \pi \cdot 10^{12}+0,6.10^{4}+\frac{225\left(e^{2}-1\right)}{34}\right) J_{0}+\frac{3 \sqrt{0.45 \cdot 10^{-6}}}{17}\left(2+6 W_{0}+0,612\right) \approx 3.768 \cdot 10^{7}
$$

It follows $\rho\left(\dot{W}^{(1)}, \dot{W}^{(0)}\right) \leq 18.84 \cdot 10^{6}$. Consequently

or

$$
\begin{gathered}
\rho\left(\left(W^{(n+1)}, J^{(n+1)}\right),\left(W^{(n)}, J^{(n)}\right)\right) \leq \frac{\left(1.13 \cdot 10^{-7}\right)^{n}}{1-1.13 \cdot 10^{-7}} 3.8 \cdot 10^{7} \quad(n=0,1, \cdots) \\
\left|\dot{W}^{(n+1)}(t)-\dot{W}^{(n)}(t)\right| \leq 7.4\left(1.13 \cdot 10^{-7}\right)^{n} 3.8 \cdot 10^{7} \leq\left(1.13 \cdot 10^{-7}\right)^{n} \cdot(2.82) \cdot 10^{8} .
\end{gathered}
$$

In the same way we can obtain an estimate for the second component of the operator $B$ :

$$
\begin{aligned}
J^{(1)}(t)= & B_{i}\left(W^{(0)}, J^{(0)}\right)(t)=\int_{T}^{t} I\left(W^{(0)}, J^{(0)}\right)(s) \mathrm{d} s-\left(\frac{t-T}{T_{0}}-\frac{1}{2}\right) \int_{T}^{T+T_{0}} I\left(W^{(0)}, J^{(0)}\right)(s) \mathrm{d} s-\frac{1}{T_{0}} \int_{T}^{T+T_{0}} \int_{T}^{t} I\left(W^{(0)}, J^{(0)}\right)(s) \mathrm{d} s \mathrm{~d} t \\
= & -W_{0} \sin \omega_{0} t+\frac{R W_{0}}{L} \int_{T}^{t} \sin \omega_{0} s \mathrm{~d} s+2 E_{0} \sqrt{L} \int_{T}^{t} \frac{e^{R / L(s-T)}}{\tilde{L}_{1}(s-T)} \mathrm{d} s \\
& +Z_{0} \int_{T}^{t} \frac{W_{0} \sin \omega_{0} s}{\tilde{L}_{1}(s-T)} \mathrm{d} s-\left(\frac{t-T}{T_{0}}-\frac{1}{2}\right)\left(2 E_{0} \sqrt{L} \int_{T}^{T+T_{0}} \frac{e^{(R / L)(s-T)}}{\tilde{L}_{1}(s-T)} \mathrm{d} s+Z_{0} \int_{T}^{T+T_{0}} \frac{W_{0} \sin \omega_{0} s}{\tilde{L}_{1}(s-T)} \mathrm{d} s\right) \\
& -\frac{1}{T_{0}} \int_{T}^{T+T_{0}}\left(2 E_{0} \sqrt{L} \int_{T}^{t} \frac{e^{(R / L)(s-T)}}{\tilde{L}_{1}(s-T)} \mathrm{d} s+Z_{0} \int_{T}^{t} \frac{W_{0} \sin \omega_{0} s}{\tilde{L}_{1}(s-T)} \mathrm{d} s\right) \mathrm{d} t .
\end{aligned}
$$




\section{Acknowledgements}

The research described in this paper was carried out within the frame work of Contract No. DUNK-01/03 -12.2009 (UNIK).

\section{References}

[1] M. Shimura, "Analysis of Some Nonlinear Phenomena in a Transmission Line," IEEE Transactions on Circuit Theory, Vol. 14, No. 1, 1967, pp. 60-68. doi:10.1109/TCT.1967.1082648

[2] L. O. Chua and P.-M. Lin, "Machine Analysis of Electronic Circuits," in Russian, Energy, Moscow, 1980.

[3] D. M. Pozar, "Microwave Engineering," 2nd Edition, New York, Wiley, 1998.

[4] P. Chirlian, "Analysis and Design of Integrated Electronic Circuits," Harper \& Row, Publishers, Inc., New York, 1987.

[5] C. R. Paul, "Analysis of Multiconductor Transmission Lines," Wiley-Interscience Publications, John Wiley \& Sons, Hoboken, 1994.

[6] L. O. Chua, C. A. Desoer and E. S. Kuh, "Linear and Nonlinear Circuits," McGraw-Hill Book Company, New York, 1987.

[7] A. H. Zemanian, "Hyperreal Waves on Transfinite, Terminated Distortionless and Lossless Transmission Lines," International Journal of Circuit Theory and Applications, Vol. 33, No. 3, 2005, pp.183-193. doi:10.1002/cta. 314

[8] S. Rosenstark, "Transmission Lines in Computer Engineering," McGraw-Hill, Inc., New York, 1994.

[9] V. G. Angelov, "Transmission Lines Terminated by Series Nonlinear RLC-Loads," Annual of the University of Architecture, Civil Engineering and Geodesy, Vol. 41, Sofia, 2000-2001, pp. 9-29.

[10] V. G. Angelov, "Lossy Transmission Lines Terminated by Nonlinear R-Loads with Exponential V-I Chara- cteristics," Journal Nonlinear Analysis, Vol. 8, No. 2, 2007, pp. 579-589.
[11] V. G. Angelov, "Periodic Regimes for Distortionless Lossy Transmission Lines Terminated by Parallel Connected RCL-Loads," In: Transmission Lines: Theory, Types and Applications. Ser. Electrical Engineering Developments, Nova Science Publishers, New York, 2011.

[12] S. Grivet-Talocia, H. M. Huang, A. E. Rudehli, F. Canavero and I. M. Elfadel, "Transient Analysis of Lossy Transmission Lines: An Efficient Approach Based on the Method of Characteristics," IEEE Transactions on Advanced Packaging, Vol. 27, No. 1, 2004, pp. 45-56. doi:10.1109/TADVP.2004.825467

[13] V. Rasvan, "Functional Differential Equations and OneDimensional Distortionless Propagation," Tatra Mountains Mathematical Publications, Vol. 43, 2009, pp. 215228.

[14] K. L. Cooke and D. W. Krumme, "Differential-Difference Equations and Nonlinear Initial Boundary Value Problems for Linear Hyperbolic Partial Differential Equations," Journal of Mathematical Analysis and Applications, Vol. 24, No. 2, 1968, pp. 372-387. doi:10.1016/0022-247X(68)90038-3

[15] A. D. Myshkis, "Linear Differential Equations with Retarded Arguments," in Russian, Nauka, Moscow, 1972.

[16] M. A. Krasnoselskii, "The Shift Operator along the Trajectories," in Russian, Moscow, 1970.

[17] Y. G. Borisovich, "Nonlinear Fredholm Mappings and Periodic Solutions of Functional Differential Equations," in Russian, Proceedings of Mathematical Faculty, Vol. 10, 1973, pp. 12-25.

[18] V. G. Angelov, "Fixed Points in Uniform Spaces and Applications," Cluj University Press, Cluj-Napoca, 2009.

[19] T. Dhaene and D. D. Zutter, "Selection of Lumped Models for Coupled Lossy Transmission Lines," IEEE Transaction on Computer-Aided Design, Vol. 11, No. 7, 1992, pp. 805-815. doi:10.1109/43.144845

[20] S. Y. Lee, A. Konrad and R. Saldanha, "Lossy Transmission Line Transient Analysis by the Finite Element Method," IEEE Transactions on Magnetics, Vol. 29, No. 2, 1993, pp. 1730-1732. doi:10.1109/20.250739

[21] Taflove and S. C. Hagness, "Computational Electromagnetics: The Finite-Difference Time-Domain Method," 2nd Edition, Artech House, Boston, 2000. 